Meta

Journal des traducteurs

Translators' Journal

\title{
La liturgie eucharistique de l'Église orthodoxe et l'histoire de sa traduction en langue française
}

\section{Felicia Dumas}

Volume 58, numéro 3, décembre 2013

URI : https://id.erudit.org/iderudit/1025050ar

DOI : https://doi.org/10.7202/1025050ar

Aller au sommaire du numéro

Éditeur(s)

Les Presses de l’Université de Montréal

ISSN

0026-0452 (imprimé)

1492-1421 (numérique)

Découvrir la revue

Citer cet article

Dumas, F. (2013). La liturgie eucharistique de l'Église orthodoxe et l'histoire de sa traduction en langue française. Meta, 58(3), 542-555.

https://doi.org/10.7202/1025050ar
Résumé de l'article

Nous nous proposons d'étudier l'histoire des différentes versions françaises du texte de la liturgie eucharistique de saint Jean Chrysostome, l'office central de l'Église orthodoxe. En principe, on peut parler de deux types de traductions qui ont circulé dans les différentes communautés orthodoxes qui sont établies en France et s’y sont développées. Nous ferons exclusivement référence à ce pays. D'une part, il y a eu des versions françaises qui accompagnaient le texte slavon surtout (mais aussi grec), qui était célébré dans des paroisses de tradition slave (ou grecque). Elles circulaient sous forme de brochures reliées, le plus souvent de façon artisanale. Leur rôle fondamental était d'accueillir linguistiquement les fidèles français qui ne parlaient pas les langues des communautés traditionnellement orthodoxes et qui participaient aux offices. D'autre part, il y a eu des versions françaises faites par des personnalités ecclésiastiques remarquables pour servir lors des célébrations dans des paroisses majoritairement francophones ou dans certaines communautés monastiques (toujours francophones). Dans ce cas, on peut parler d'un rôle liturgique-rituel par excellence de ces traductions, le texte de la divine liturgie ayant été traduit explicitement pour permettre sa célébration en français.
Ce document est protégé par la loi sur le droit d'auteur. L'utilisation des services d’Érudit (y compris la reproduction) est assujettie à sa politique d'utilisation que vous pouvez consulter en ligne.

https://apropos.erudit.org/fr/usagers/politique-dutilisation/ 


\title{
La liturgie eucharistique de l'Église orthodoxe et l'histoire de sa traduction en langue française
}

\author{
FELICIA DUMAS \\ Université Alexandru Ioan Cuza, Iași, Roumanie \\ felidumas@yahoo.fr
}

\begin{abstract}
RÉSUMÉ
Nous nous proposons d'étudier l'histoire des différentes versions françaises du texte de la liturgie eucharistique de saint Jean Chrysostome, l'office central de l'Église orthodoxe. En principe, on peut parler de deux types de traductions qui ont circulé dans les différentes communautés orthodoxes qui sont établies en France et s'y sont développées. Nous ferons exclusivement référence à ce pays. D'une part, il y a eu des versions françaises qui accompagnaient le texte slavon surtout (mais aussi grec), qui était célébré dans des paroisses de tradition slave (ou grecque). Elles circulaient sous forme de brochures reliées, le plus souvent de façon artisanale. Leur rôle fondamental était d'accueillir linguistiquement les fidèles français qui ne parlaient pas les langues des communautés traditionnellement orthodoxes et qui participaient aux offices. D'autre part, il y a eu des versions françaises faites par des personnalités ecclésiastiques remarquables pour servir lors des célébrations dans des paroisses majoritairement francophones ou dans certaines communautés monastiques (toujours francophones). Dans ce cas, on peut parler d'un rôle liturgique-rituel par excellence de ces traductions, le texte de la divine liturgie ayant été traduit explicitement pour permettre sa célébration en français.
\end{abstract}

\section{ABSTRACT}

This paper purports to study the history of the various translations in the French language of the Eucharistic Liturgy of Saint John Chrysostomos, the main religious service in the Orthodox Church. Roughly speaking, there are two types of translations that circulated amongst the various Orthodox communities which settled and developed in France, because this paper will approach only the Metropolitan France. On the one hand, there used to be French translations accompanying the Slavonic (and also Greek) text, which lay at the basis of the religious service in the parishes of Slavic (or Greek) tradition. As a rule, these would circulate as hand-made stitch books and their main role was to accommodate linguistically the French-speaking parishioners who could not speak the languages of the traditionally Orthodox communities whose services they attended. On the other hand, there were also translations by outstanding members of the clergy to celebrate them in mostly French-speaking parishes or in certain (also French-speaking) monastic communities. In this case, we may speak about a liturgical-ritualistic role par excellence of these translations, the text of the Holy Liturgy being translated with the explicit purpose of its use in French.

\section{MOTS-CLÉS/KEYWORDS}

liturgie, Église orthodoxe, diachronie, contexte culturel

liturgy, Orthodox Church, diachronic study, cultural context

\section{Introduction : la liturgie eucharistique au cœur de l'Église orthodoxe}

La liturgie eucharistique constitue l'office central, le plus important de l'Orthodoxie. Elle est célébrée par un ministre consacré, un évêque (un archevêque, un métropolite 
ou un patriarche) ou un prêtre. Dans le premier cas, on parle de liturgie pontificale. Elle porte le qualificatif d'eucharistique, parce qu'elle réside dans la célébration de l'eucharistie, «fondement de l'Église et sacrement par excellence. Le mot eucharistie vient du grec et signifie "action de grâces". Par la liturgie eucharistique, l'homme rend grâce à Dieu, et le remercie pour toutes choses ${ }^{1}$ ». Pour les croyants, pendant la célébration, au cours de l'anaphore, le pain et le vin (eucharistiques) sont transformés, par l'action du Saint-Esprit invoqué par le ministre, en corps et en sang du Christ auxquels les fidèles communient pour la rémission de leurs péchés et la vie éternelle.

L'Église orthodoxe connaît deux liturgies eucharistiques, attribuées aux saints Jean Chrysostome et Basile le Grand de Césarée. La première est célébrée plus souvent pendant l'année liturgique que la deuxième. C'est la raison pour laquelle nous nous rapporterons plus loin à ses versions en langue française, en précisant chaque fois si celles-ci ont été accompagnées ou non des traductions de la liturgie de saint Basile de Césarée.

La liturgie eucharistique attribuée à saint Jean Chrysostome fait partie de la famille des liturgies syro-antiochiennes ${ }^{2}$. Elle est composée de trois grandes parties: la partie introductive appelée proscomidie ou office de la prothèse (Dumas 2010a: 248) (pendant laquelle le ministre prépare les dons, le pain et le vin, qui vont être consacrés), la liturgie des catéchumènes ou de la parole (qui réside dans toute une série de prières, de doxologie et d'intercession, ainsi que dans l'écoute de la parole divine, par la lecture de l'évangile) et la liturgie des fidèles (centrée sur la consécration eucharistique et la communion) (Dumas 2000: 66). Par la célébration de la liturgie eucharistique, la communauté des fidèles participants actualise, à l'endroit en question, l'Église du Christ:

[L]'Eucharistie fait l'Église. Celle-ci n'est rien d'autre que le corps glorifié du Christ uni à ses membres [...]. Cette Église-Corps du Christ est rendue présente ici-bas partout où existe une communauté de chrétiens qui se rassemblent, dans une ville ou une bourgade, autour de leur évêque ou de l'un de ses prêtres qui le représentent, et reçoit de sa main le corps eucharistique du Seigneur. (Deseille 2005: 20)

L'eucharistie représente aussi l'un des sept sacrements de l'Église, qui se trouvent au centre même de la vie de celle-ci et qui visent, pour les croyants, à la sanctification de l'homme. D'où l'importance fondamentale de la célébration eucharistique pour l'existence même de l'Église. Dans les Églises orthodoxes répandues dans le monde, la célébration liturgique se fait (en général, sauf pour le slavon) dans la langue du peuple qui participe à cet office pour qu'elle puisse être comprise. C'est ce qui se passe aussi en France depuis l'implantation de l'Orthodoxie sur son territoire.

\section{L'Église orthodoxe en France et ses besoins linguistiques de célébration}

L'Église orthodoxe a commencé à s'enraciner en France ${ }^{3}$ au début du siècle dernier (certains disent même à la fin du XIX ${ }^{\mathrm{e}}$ siècle), notamment par l'intermédiaire de deux grandes vagues d'émigration, russe (après la Révolution de 1917) et grecque (surtout après 1922), suivies un peu plus tard, après la chute du communisme dans les pays de l'Europe de l'Est, par d'autres: roumaine, serbe, bulgare, moldave (Dumas 2009). Au début, dans la plupart des communautés ainsi créées, les offices orthodoxes ont été célébrés dans les langues d'origine de ces migrants ${ }^{4}$. Ensuite, deux phénomènes se sont produits. D’une part, de plus en plus de Français ont intégré ces communau- 
tés organisées entre-temps en paroisses, qui sont devenues ainsi des paroisses francophones, et d'autre part, la deuxième génération de ces migrants parlait davantage le français que sa langue d'origine au fil de son intégration dans la société française. Le hiéromoine Samuel, auteur de l'Annuaire de l'Église Orthodoxe de France, parle en ce sens de deux types de besoins linguistiques concernant l'emploi du français comme langue liturgique de l'Église orthodoxe: «besoins pastoraux des fidèles français, mais aussi des enfants et des petits-enfants d'immigrés, de plus en plus intégrés à la société française» (Samuel 2010: $8^{5}$ ). Il s'agit d'une intégration comprise surtout dans le sens de l'emploi exclusif du français en contexte social, scolaire et professionnel et de la conservation de la langue d'origine (au mieux) dans les contextes familiaux et la vie personnelle.

La principale raison d'être des traductions françaises du texte de la liturgie eucharistique a été la compréhension de ce dernier par les fidèles francophones (ainsi que par cette deuxième génération de migrants) pendant sa célébration. À ce titre, on peut parler de deux types de versions qui ont circulé dans les différentes communautés orthodoxes qui se sont établies et développées en France, car nous nous intéressons exclusivement à l'Hexagone dans le présent article. D'une part, il y a eu des traductions françaises qui accompagnaient le texte slavon surtout (mais aussi grec) utilisé dans des paroisses de tradition slave (ou grecque). Elles circulaient en général sous forme de brochures reliées, le plus souvent de façon artisanale et parfois même polycopiées. Leur rôle fondamental était donc d'accueillir linguistiquement les fidèles français qui ne comprenaient pas les langues liturgiques des communautés traditionnellement orthodoxes avec lesquelles ils participaient aux offices, en l'occurrence le slavon et le grec. D'autre part, il y a eu des versions françaises de la liturgie eucharistique de saint Jean Chrysostome faites par des personnalités ecclésiastiques remarquables, utilisées dans des paroisses majoritairement francophones ou dans certaines communautés monastiques (en principe, de juridictions différentes). Dans ce cas, on peut parler d'un rôle liturgique-rituel par excellence de ces traductions, les textes des divines liturgies étant traduits dans le but explicite (ou explicité par le contexte culturel de la traduction) de leur célébration en français. Parmi ce deuxième type de versions françaises, on rencontre des textes publiés par des maisons d'éditions religieuses (ou les éditions de certains monastères), ainsi que des brochures à circulation restreinte, destinées à quelques communautés - paroissiales ou monastiques - très précises (qui ont précédé les premières, dont elles constituaient les ébauches, le point de départ). On pourrait donc parler de traductions faites pour l'usage liturgique immédiat (dans le sens de leur nécessité) par certaines communautés, qui ont circulé sous forme de brochure, et les traductions officielles et officialisées, publiées dans certaines juridictions, avec la bénédiction des autorités ecclésiastiques, des hiérarques, ou autres fédérations (comme l'Assemblée des évêques orthodoxes de France). Nous analyserons par la suite leur cohabitation (plutôt harmonieuse), ainsi que leur concurrence cultuelle et culturelle, en principe juridictionnelle. Nous utiliserons comme méthode de recherche l'investigation historique sur corpus. Le corpus sera représenté par l'ensemble des traductions françaises de la liturgie eucharistique de saint Jean Chrysostome qui ont circulé en France, sous forme de publications ou de brochures reliées, étant utilisées rituellement dans les différentes communautés orthodoxes implantées sur le territoire, depuis les origines de l'Orthodoxie d'expression française jusqu’à présent. 


\section{La liturgie de saint Jean Chrysostome et ses différentes traductions françaises}

L'histoire des traductions françaises ${ }^{6}$ du texte de la liturgie de saint Jean Chrysostome s'inscrit dans une chronologie d'assez longue durée, qui remonte au XIX ${ }^{e}$ siècle. La première version française de cette liturgie eucharistique a été réalisée par un prêtre catholique français, originaire de Blois, converti à l'Orthodoxie: l'archimandrite Wladimir Guettée (1816-1892). Après son entrée dans l'Église orthodoxe, il lui a fallu traduire en français le texte liturgique fondamental de sa pratique, qu'il devait célébrer en tant que ministre consacré, soit celui de la liturgie eucharistique de saint Jean Chrysostome, selon les affirmations de plusieurs fidèles orthodoxes français que nous avons recueillies lors de quelques enquêtes. On ne sait pas ce qu'est devenue sa traduction française, qui a dû circuler, sans doute, sous forme de brochure imprimée et reliée de façon artisanale.

Toujours sous l'angle chronologique, une autre traduction en langue française du texte de la liturgie eucharistique de saint Jean Chrysostome a été faite par Dom Placide de Meester, qui l'a publiée en 1925 à côté du texte grec original dans une édition bilingue (Saint Jean Chrysostome $\left.\mathrm{IV}^{\mathrm{e}} \mathrm{s} . / 1925\right)^{7}$. La motivation de cette traduction différait de la précédente. L’auteur voulait accompagner, pour ses lecteurs français, le texte grec établi de cette liturgie eucharistique. Il ne s'agissait donc pas d'une traduction à usage liturgique, mais d'une démarche culturelle, érudite, d'initiation des lecteurs français à la beauté du texte de cette liturgie eucharistique, rendue accessible par sa traduction française.

La première église orthodoxe de France a été la cathédrale saint Alexandre Nevsky de Paris (consacrée en 1861), à présent siège de l'Archevêché des Églises russes en Europe occidentale, dans la juridiction du Patriarcat œcuménique de Constantinople. La première paroisse francophone a été créée dans la même ville en 1927 et confiée au père Lev Gillet. Celui-ci a aussi traduit la liturgie de saint Jean Chrysostome en langue française pour s'en servir dans les célébrations. Aux dires du père archimandrite Placide Deseille, la version française de Gillet a également été éditée par Dom Placide de Meester (grand connaisseur de la richesse et de la beauté liturgique des textes byzantins) et reproduite dans l'ouvrage de Paul Evdokimov (1985). Cette traduction a servi de point de repère français au père Placide Deseille lorsqu'il a luimême entrepris la traduction française de la Divine liturgie de notre saint Père Jean Chrysostome, publiée sous forme de brochure non datée au Monastère Saint-AntoineLe-Grand, métochion de Simonos Petra, dont il est le supérieur (ou l'higoumène) ${ }^{8}$. Le bref «avertissement» qu'il formule en avant-propos de sa version française apporte déjà des précisions quant à sa spécificité (« usage athonite» de la source, car conforme au statut du monastère-métochion, dépendant d'un autre grand monastère, du Mont Athos) et oriente le lecteur vers la traduction officiellement publiée en 2009: «La présente traduction a été faite sur le texte grec publié dans le Hiératikon édité par le Monastère de Simonos Petra, Hagion Oros, 1992. Elle suit par conséquent les usages

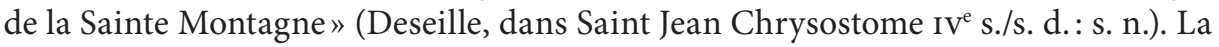
version publiée en 2009 (avec ISBN) aux éditions des deux monastères-métochia du monastère athonite de Simonos Petra fondés par le père archimandrite Placide Deseille en France n'a plus d'avant-propos ni d'autre appareil paratextuel introductifexplicatif ${ }^{9}$. Elle comporte une seule précision, de grande importance toutefois, écrite 
en guise de sous-titre: «selon l'usage du Mont Athos» (Saint Jean Chrysostome et Saint Basile de Césarée iv ${ }^{\mathrm{e}}$ s./2009: $3^{10}$ ). Le traducteur attire ainsi l'attention (toujours de façon paratextuelle) sur les particularités rituelles de cette traduction de la liturgie qui, individualisées dans les rubriques, sont révélatrices de son usage très précis, athonite. Il ne mentionne plus la source de la traduction ${ }^{11}$, ainsi devenue implicite. Nous y reviendrons.

En 1965 paraissait à Nice une traduction française attribuée à Mgr Sylvestre, évêque vicaire de l'Archevêché des Eglises orthodoxes russes en Europe occidentale, exarchat du Patriarcat œcuménique. Elle a été imprimée d'abord sous forme de brochure, comme nous avons pu l'apprendre de deux sources d'information différentes: la recension faite par Jean-Claude Larchet ${ }^{12}$ de la traduction susmentionnée des liturgies eucharistiques par l'archimandrite Placide Deseille (Larchet considère la version de Mgr Sylvestre comme la première version française de la liturgie de saint Jean Chrysostome) ${ }^{13}$, et les informations de quelques responsables laïcs des paroisses de cet archevêché, qui se souviennent encore de cette publication. D’après les mêmes fidèles orthodoxes (qui dépendent donc de cet archevêché), Mgr Sylvestre n'avait luimême pas les compétences nécessaires en langue française pour traduire seul le texte de la divine liturgie. Il n'a fait que bénir le travail des traducteurs. Ceux-ci étaient en fait les prêtres (et théologiens) orthodoxes Jean Meyendorff et Boris Bobrinskoy, tous deux d'origine russe. Au départ, selon les témoignages des mêmes fidèles orthodoxes français, cette version française accompagnait le texte slavon de la liturgie. Quand les auteurs ont voulu se détacher petit à petit de l'original slavon pour que la version française puisse être employée indépendamment de celui-ci, en toute autonomie linguistique, les traducteurs se sont rendu compte des nombreuses imperfections de leur travail et ont révisé leur première traduction. La motivation de ce travail de correction était essentiellement pratique, motivée par un souci de fidélité rituelle (discursive et musicale) du texte, qui devait servir pour la célébration. Le résultat de leurs efforts a été publié en 1981, aux éditions Liturgica, mais toujours accompagnée du texte slavon, dans une édition bilingue ${ }^{14}$. Une autre source confirme la reprise de la version de Nice de 1965 pour cette retraduction de 1981: le passionnant (et très érudit) livre d'herméneutique de la liturgie eucharistique byzantine signé par Henryk Paprocki, professeur de théologie au Séminaire orthodoxe de Varsovie (Paprocki 1993, traduit par Lhoest: 405). Nous pensons que, dans ce cas très précis, vu les efforts des traducteurs pour améliorer la version française, la publication de celle-ci en édition bilingue avec le texte slavon a joué un rôle de légitimation de l'usage du français en tant que langue liturgique de l'Orthodoxie, et de la traduction française comme conforme à l'original, ayant toutes les qualités requises pour être célébrée indépendamment du texte slavon, en toute autonomie. La traduction française de la liturgie de saint Jean Chrysostome n'a donc plus la fonction d'accompagnement du texte slavon, mais elle s'affirme en tant que texte liturgique autonome et indépendant, authentiquement orthodoxe, car conforme au texte slavon qui l'accompagne, qui est lui, traditionnellement orthodoxe.

Dans la diachronie, une autre version française de la liturgie eucharistique de saint Jean Chrysostome a été faite par le père Denis Guillaume, ancien moine grécocatholique (au moment de la traduction) converti à l'Orthodoxie vers la fin de sa vie. Excellent connaisseur de la langue grecque, le père Denis Guillaume est, sans aucun doute, le traducteur le plus prolifique des textes et des livres liturgiques de l'Église 
orthodoxe. Il a traduit en français l'ensemble des offices liturgiques: les liturgies eucharistiques, la Grande et la Petite Pannychide, le Grand Euchologe et l'Arkhiératikon, l'office pour les professions monastiques, le Triode de Carême, et une partie des ménées russes, grecques, roumaines et serbes. En même temps, il a composé (toujours en français) de nombreux acathistes pour des saints ayant vécu en France. La plupart de ces traductions sont parues aux éditions du monastère de Chevetogne (en Belgique) ou à la Diaconie Apostolique. La version française de la liturgie de saint Jean Chrysostome, qui nous intéresse ici, a été publiée dans un petit livre de poche qui regroupe les trois liturgies, soit celles de saint Jean Chrysostome, de saint Basile le Grand, ainsi que la liturgie des présanctifiés ${ }^{15}$. Même si le nom du traducteur n'y est pas mentionné, il est suggéré par le lieu de la publication: Diaconie Apostolique, Rome. «Diaconie Apostolique» est le nom d'une institution créée par le père Denis Guillaume, dont il a été le seul membre. Le nom de venait du fait qu'il était diacre du siège apostolique de Rome. Avant d'avoir été reçu dans l'Orthodoxie en 1994 (dans l'Eglise orthodoxe de Finlande), il a été, comme nous l'avons déjà dit, moine au monastère de Chevetogne, rattaché temporairement au Collège grec de Rome. Le père archimandrite Placide Deseille le confirme dans un entretien sur les particularités de l'orthodoxie d'expression française, réalisé en 2009: «La Diaconie Apostolique est un nom, mais en fait il ne s'agit que d'une seule personne; c'est le père Denis Guillaume qui a fait ces traductions, qui a tout fait» (Dumas 2009: 12). Ayant été publiée dès 1986, à un moment où il n'y avait pas beaucoup de versions françaises de la liturgie de saint Jean Chrysostome (à part celle des éditions Liturgica, déjà mentionnée), sa traduction a connu une assez grande circulation en France, dans plusieurs paroisses francophones, tout comme dans de nombreux monastères:

Ce sont des traductions adoptées par certains, mais cela n'a aucun rôle officiel dans l'orthodoxie. [...] Au fond, on s'en sert surtout dans des monastères, parce que c'est le seul moyen d'avoir une traduction de la totalité des livres liturgiques. Dans les paroisses, où ils ont toujours des offices abrégés, seulement le dimanche, [...] n'ayant pas beaucoup de choix, ils se servent de la traduction du père Denis Guillaume. (Dumas 2009: 12)

On remarque donc que la publication de cette traduction française comblait une lacune relativement aux textes liturgiques fondamentaux, dont les paroisses francophones avaient fortement besoin. La traduction parue en 1981, aux éditions Liturgica, était perçue comme de tradition russe, et trop marquée du point de vue juridictionnel. La version du père Denis Guillaume avait le mérite d'être publiée par une institution non marquée de ce point de vue (d'une appartenance juridictionnelle orthodoxe précise) et de jouir d'une assez grande circulation. Malgré cela, pour certains, elle avait un défaut majeur: elle n'était pas l'œuvre d'un orthodoxe, tout excellent traducteur du grec vers le français qu'il soit ${ }^{16}$ !

Trois autres versions françaises de la même liturgie de saint Jean Chrysostome allaient paraître en France après l'an 2000, dont une en deux éditions. La première est une traduction faite en milieu monastique par des moines français avec de très bonnes compétences en langue grecque: l'archimandrite Jacob, le hiéromoine Elisée et le père dr. Y. Goldman, du monastère de Cantauque, francophone, dépendant du point de vue canonique de la Métropole orthodoxe roumaine d'Europe occidentale et méridionale. La version française de la liturgie eucharistique de saint Jean Chrysostome est accompagnée dans cette édition de celle de l'autre liturgie eucharistique de l'Église 
orthodoxe, attribuée à saint Basile le Grand, de Césarée (Saint Jean Chrysostome $\mathrm{IV}^{\mathrm{e}}$ s./2002) ${ }^{17}$. La seconde édition de ces deux liturgies a été publiée en 2006 (Saint Jean Chrysostome IV $\left.{ }^{\mathrm{e}} \mathrm{s} . / 2002 / 2006\right)^{18}$ et a rendu la première extrêmement rare et très difficile à trouver. Grâce à l'amabilité du père archimandrite Jacob, higoumène du monastère, nous sommes en possession de la version électronique de celle-ci, dont nous avions grand besoin afin de pouvoir comparer les textes des deux éditions et d'étudier les corrections faites dans la seconde par rapport à la première. Tout d'abord, nous avons remarqué l'humilité des traducteurs, exprimée discursivement dans l'appareil paratextuel de leur première version française, où ils se déclarent explicitement ouverts et réceptifs à toute remarque et observation de la part des lecteurs-bénéficiaires (liturgiquement) de leur traduction: «Les suggestions en vue d'une édition améliorée et plus complète seront reçues avec reconnaissance» (Les traducteurs, dans Saint Jean Chrysostome et Saint Basile de Césarée IV s./2002). Conscients de l'importance liturgique et culturelle-confessionnelle de leur démarche, ils se montrent ainsi soucieux de la qualité de leur traduction, jugée perfectible. En même temps, ils précisent scrupuleusement (du point de vue scientifique) les manuscrits grecs à partir desquels ils ont traduit les textes des deux liturgies eucharistiques en langue française:

Cette traduction des Divines Liturgies de saint Jean Chrysostome et de saint Basile a été faite à partir des textes grecs édités par Brightman (Oxford, Clarendon Press, 1896). Par endroit cependant, les leçons du manuscrit Barberini 355 ont été préférées. Les psaumes et les versets psalmiques sont, en général, une adaptation d'une traduction de la Septante. (Les traducteurs, dans Saint Jean Chrysostome et Saint Basile de Césarée IV $V^{\mathrm{e}}$ s./2002: s.n.; voir note 17 )

Ces indications des sources de la traduction sont gardées telles quelles dans la seconde édition de leur version française, parue quatre ans plus tard (voir note 18). Comme nous l'avons déjà dit, nous étions très intéressée par l'analyse des corrections éventuelles apportées au texte de la première version, quelle qu'en soit la nature. Après avoir comparé minutieusement les deux éditions, nous n'avons remarqué qu'une seule différence notable relative au texte de l'Hymne à la Mère de Dieu (appelée aussi l'Axion estin, notamment dans la tradition grecque, ou bien Il est digne (Dumas 2010a: 70), légèrement différent dans la seconde édition du Liturgikon ${ }^{19}$. Les modifications de la première variante de cette hymne visent deux aspects principaux: la musicalité du texte (qui doit être chanté pendant la célébration de la divine liturgie) et la non-ambiguïté dogmatique de certains termes qui s'y retrouvent. Le premier a été résolu par une simplification phrastique, doublée d'une traduction différente, explicitée: ainsi, le début de cette hymne «Il est digne en vérité de te louer/ toi qui enfantes Dieu» (Saint Jean Chrysostome ( $\mathrm{IV}^{\mathrm{e}}$ s./2002: 3 ; voir note 17) est devenu «Il est digne en vérité de te dire bienheureuse» (Saint Jean Chrysostome (IV ${ }^{e}$ s./2002/2006: 52 ; voir note 18), et la séquence centrale «Toi qui es plus vénérable/ que les chérubins/ et incomparablement plus glorieuse/ que les séraphins» (Saint Jean Chrysostome (IV s./2002: 3) a été allégée de sa subordonnée relative sous la forme «Plus vénérable que les chérubins/ et incomparablement plus glorieuse/ que les séraphins » (Saint Jean Chrysostome (IV s./2002/2006: 52), qui rythmait autrement, du point de vue de la musicalité, cette hymne. Le deuxième aspect a été réglé par la substitution du déterminant toute immaculée (de la première version), employé à l'égard de la Mère de Dieu, par le syntagme toute irréprochable, que l'on retrouve aussi dans la traduction 
française en usage dans les monastères fondés par le père archimandrite Placide Deseille, déjà mentionnée plus haut. Sur le plan lexico-grammatical, ce dernier syntagme est construit sur le même modèle que le premier (par préfixation négativeprivative à valeur de superlatif axiologique); il est totalement neutre du point de vue des connotations théologiques; en revanche, le déterminant toute immaculée employé dans la première version de cette hymne risquait de prêter à confusion par la forme même de son signifiant, qui pouvait être mis en relation avec le dogme catholique de l'immaculée conception, non reconnu et rejeté par l'Église orthodoxe. Cette version française de la liturgie eucharistique de saint Jean Chrysostome (accompagnée de la liturgie de saint Basile le Grand) est employée notamment dans le monastère où elle a été publiée, ainsi que dans quelques paroisses francophones qui dépendent aussi de la Métropole orthodoxe roumaine d'Europe occidentale et méridionale. C'est pourquoi nous pensons que la raison principale de sa publication a été d'ordre pratique, pour l'usage liturgique. Les moines de Cantauque (certainement pas tout à fait satisfaits des autres versions françaises déjà existantes) ont voulu disposer de leur propre traduction de la liturgie eucharistique, dans le but pratique de pouvoir la célébrer dans leur communauté.

En 2007 était publiée une autre traduction française de la même liturgie de saint Jean Chrysostome, par la Fraternité orthodoxe en Europe occidentale, avec la bénédiction de l'Assemblée des évêques orthodoxes de France (Saint Jean Chrysostome $\left.\mathrm{IV}^{\mathrm{e}} \mathrm{s} . / 2007\right)^{20}$. Il s'agit d'une édition où le texte de cette liturgie n'est plus accompagné de celui de la liturgie de saint Basile de Césarée. Elle bénéficie d'un appareil paratextuel riche et complexe, ce qui témoigne d'une véritable réflexion dans ce sens de la part des traducteurs. Mais qui est donc la Fraternité orthodoxe en Europe occidentale, l'éditeur de cette traduction? C'est un organisme constitué de croyants orthodoxes, de clercs et de fidèles, de différentes juridictions et de divers pays occidentaux, animés par le désir de «témoigner de l'unité de l'Église» et de «remédier aux inconvénients engendrés par la multiplicité des juridictions», qui œuvrent donc, sous l'omophore de leurs évêques (canoniques) respectifs, pour l'implantation en Europe occidentale d'une vie ecclésiale unifiée ${ }^{21}$. Cette Fraternité propose, entre autres, des versions françaises des textes liturgiques - à valeur «universellement acceptable» dans le monde orthodoxe occidental -, dont celle que nous venons de mentionner. Cette édition de la liturgie de saint Jean Chrysostome est précédée d'une préface du théologien orthodoxe Nicolas Lossky, président de la Commission liturgique de l'Assemblée des évêques orthodoxes de France (AEOF), qui légitime (en tant qu'autorité ecclésiastique hiérarchique), par son accord (et sa bénédiction), la traduction française du texte proposée par le groupe des traducteurs «agréés» par la Fraternité orthodoxe $^{22}$. D’ailleurs, la préface est suivie d'un liminaire, signé par les membres de l'AEOF (tous les évêques orthodoxes canoniques de France), où est expliqué le rôle unificateur de cette assemblée, qui chapeaute les différentes juridictions orthodoxes présentes en Europe occidentale. Elle ne fait toutefois aucune référence au texte source de la traduction ni aux manuscrits à partir desquels les traducteurs ont accompli leur version française, ni aux autres traductions du même texte de la liturgie de saint Jean Chrysostome qui existent en français. La préface insiste beaucoup sur les qualités de conformité liturgique de cette version, garanties et légitimées par l'autorité compétente en la matière, la Commission liturgique de l'AEOF, directement engagée dans l'acte de la traduction: "Soulignons que cette traduction de base [proposée par le 
groupe de traduction de la Fraternité orthodoxe] a été revue et passée au crible par la Commission liturgique, souvent avec la participation d'évêques» (Lossky 2007: 5 dans Saint Jean Chrysostome IV s./2007; voir note 20). Les seules remarques traductologiques de la préface concernent une version française du Notre Père, appelée «traduction commune à tous les chrétiens francophones", proposée dans les années soixante par des théologiens catholiques, orthodoxes et protestants, considérée comme inappropriée, car inexacte (sans pour autant que nous soient fournis les détails de cette inexactitude): «Elle pose en effet un certain nombre de problèmes de traduction qu'il serait souhaitable d'examiner avec toute l'attention qui s'impose» (Saint Jean Chrysostome IV s./2007: 84). Les traducteurs proposent de la remplacer par une nouvelle traduction, insérée timidement dans la préface (Lossky 2007: 6 dans Saint Jean Chrysostome IV s./2007; voir note 20) ainsi que dans la version française intégrale de la liturgie eucharistique de saint Jean Chrysostome, en notes en bas de pages (Saint Jean Chrysostome IV $\mathrm{IV}^{\mathrm{e}}$ s/2007: 84). L'unicité de cette remarque concernant le changement apporté aux versions antérieures du texte liturgique (qui inclut celui de l'oraison dominicale) pourrait nous faire croire que cette nouvelle traduction de la liturgie n'a été faite, en quelque sorte, que pour légitimer la démarche de proposer une version différente du Notre Père, dans le cadre de ce contexte liturgique large de sa pratique. Plusieurs de nos informateurs orthodoxes nous ont avoué que sa circulation dans le milieu orthodoxe français est plutôt restreinte, et qu'elle n'est pas vraiment utilisée pour la célébration.

La plus récente des traductions françaises de la divine liturgie de saint Jean Chrysostome (et, par conséquent, la dernière dans la diachronie) a été publiée en 2009 aux éditions des monastères Saint-Antoine-Le-Grand et de Solan, dans un Liturgikon qui comprend aussi les textes de la liturgie eucharistique de saint Basile le Grand (ou de Césarée) et de la liturgie des dons présanctifiés (Saint Jean Chrysostome $\mathrm{IV}^{\mathrm{e}}$ s./2009) ${ }^{23}$. Même si le nom du traducteur n'est pas mentionnée ${ }^{24}$, le lieu de publication de cette traduction le désigne implicitement: il s'agit très probablement du père archimandrite Placide Deseille, fondateur des deux monastères et higoumène du premier. Le sous-titre, "selon l'usage du Mont Athos», suggère le but de cette traduction, qui est celui de servir pour le propre usage liturgique des deux monastères français, qui respectent le typikon athonite, du fait de leur particularité fondamentale, d'être des métochia français (des monastères-dépendants) du monastère athonite de Simonos Petra. Cette version, très vraisemblablement réalisée par le père archimandrite Placide Deseille, grand connaisseur du grec (dont il a traduit en français de nombreux textes patristiques; Dumas 2010a), a été très appréciée par l'un des plus grands théologiens français contemporains, Jean-Claude Larchet, dans une recension publiée sur le site orthodoxie.com. Jean-Claude Larchet y condamne la prise de position de l'un des membres de la Commission liturgique de l'AEOF, qui, tout de suite après la parution de cette nouvelle version, s'est plaint auprès de l'AEOF de la prolifération des traductions liturgiques en France. Tout en insistant sur les qualités linguistiques et stylistiques de la traduction, Jean-Claude Larchet dénonce explicitement le manque de compétences en grec liturgique des traducteurs de cette commission liturgique et, implicitement, leur interprétation de la coexistence des différentes versions françaises de la liturgie de saint Jean Chrysostome en termes de concurrence menaçante. 


\section{En guise de conclusion}

L'histoire riche et passionnante des traductions françaises de la liturgie eucharistique de saint Jean Chrysostome s'avère être en même temps une histoire retraductive des plus intéressantes, caractérisée par une coexistence harmonieuse sur l'ensemble, ou en tout cas, non conflictuelle de ces différentes traductions liturgiques. Ces versions ont été motivées au départ par des besoins pastoraux explicites, accomplissant un rôle d'accompagnement d'un autre texte rédigé dans une langue traditionnellement orthodoxe (en général le slavon), ou en tant que traductions liturgiques autonomes utilisées lors des célébrations dans des communautés francophones de différentes juridictions. En même temps, ces traductions ont été sous-tendues aussi par des raisons d'amélioration linguistique (dans le sens de la clarté et de la non ambiguïté) ou de précision d'un usage particulier (en principe, monastique). Elles sont non seulement des produits cultuels, légitimés par l'autorité religieuse reconnue de leurs auteurs, mais aussi des produits culturels, inscrits dans une diachronie plutôt significative, qui s'étend de 1965 jusqu'en 2009. Cette diachronie doit être comprise justement dans ce sens, culturel, et dans la direction de Benveniste, comme «une succession de synchronies" (Benveniste 1966: 12), caractérisée chacune par tout un ensemble de facteurs déterminants, que nous avons essayé d'étudier ici: la juridiction, la personnalité ecclésiastique, le profil linguistique et les motivations personnelles ou communautaires des traducteurs.

Pour ces raisons de spécificité cultuelle et culturelle du texte traduit, nous avons préféré interpréter ainsi la réalité complexe de l'histoire de ces traductions en langue française, qui pourrait être envisagée aussi comme une histoire retraductive. Effectivement, d'un certain point de vue et dans un sens large, c'est d'une succession de retraductions de la liturgie eucharistique de saint Jean Chrysostome qu'il s'agit. Toutefois, le phénomène retraductif est considéré par la plupart des traductologues comme étant caractéristique plutôt du texte littéraire, qui peut subir d'innombrables traductions. Nous mentionnons dans ce sens une affirmation d'Enrico Monti que nous partageons, selon laquelle «la littérature est le domaine où le retraduction trouve son expression la plus vitale» (Monti 2012: 12). D’un autre côté, un auteur comme Meschonnic parle de retraduction aussi et surtout dans le cas de la Bible: «Traduire la Bible, plus que tout autre texte encore, étant donné l'histoire des effets de la Bible, est un retraduire» (Meschonnic 1999: 436). Il insiste beaucoup sur la poétique du rythme et sur les particularités de l'acte de traduire le rapport du divin dont il est question dans la Bible.

Dans l'acception de variante nouvelle de traduction littéraire, la retraduction est engendrée par des contraintes socioculturelles, qui influencent l'évolution de la langue-cible, qui est aussi une langue-culture d'arrivée. Cette remarque est valable également pour les retraductions de la Bible, qui sont engendrées par ce que Meschonnic appelle la «critique» qu'elles imposent (Meschonnic 1999: 436). De leur côté, les différentes traductions françaises du texte de la liturgie eucharistique de saint Jean Chrysostome mentionnées dans le présent travail ont contribué de manière essentielle à la formation, par individualisation lexicale normative, de la terminologie religieuse orthodoxe en langue française, qui se devait et se voulait être une terminologie spécialisée. On peut remarquer dans ce sens une influence claire exercée par certaines de ces versions sur l'imposition de plusieurs normes lexicales de cette 
terminologie orthodoxe, comme pour le terme irréprochable, par exemple, qui a remplacé celui d'immaculée, en tant que déterminant de la Mère de Dieu dans l'hymne liturgique qui lui est consacrée ${ }^{25}$. Ce qui prouve en même temps que la traduction interlinguale est « en réalité seconde par rapport à la traduction intralinguale [...], sans laquelle toute compréhension serait impossible» (Oustinoff 2011: 9). À travers cette terminologie, le français s'avère être une langue d'accueil de la culture et de la pratique liturgique orthodoxes, en tant que support d'expression des contenus confessionnels de l'Église orthodoxe, largement représentés jusque là comme «orientaux». De plus, cette terminologie religieuse orthodoxe participe, au niveau des macro-contextes discursifs religieux, dont les traductions des liturgies eucharistiques font partie, ainsi que des célébrations rituelles proprement dites, à l'individualisation d'une identité confessionnelle de l'Orthodoxie en milieu culturel et linguistique français (Dumas 2011: 58).

Sans avoir vraiment la conscience de prendre part à un ample phénomène retraductif, les traducteurs des différentes versions françaises de la liturgie eucharistique de saint Jean Chrysostome ont agi pour la nécessité linguistico-rituelle de la pratique liturgique proprement dite de l'Église orthodoxe en France (compte tenu de ses particularités, dont la coexistence sur son territoire de plusieurs juridictions canoniques). Autrement dit, même s’il étaient préoccupés par la question du «comment traduire» (Pym 1997), ces traducteurs étaient surtout animés par l’impératif du «il faut traduire», pour les raisons déjà mentionnées.

\section{NOTES}

1. Anonyme (1985): Vocabulaire théologique orthodoxe. Paris: Cerf, 125.

2. «La liturgie de saint Jean Chrysostome est sans doute une ancienne liturgie d'Antioche, retouchée par ce saint et introduite par lui à Constantinople quand il devint archevêque de cette ville» (Deseille 2005: 4). Voir aussi, traduit par Lhoest, Paprocky (1993: 73).

3. Pays occidental communément représenté comme «majoritairement» catholique.

4. Nous employons ici le terme dépourvu de toute connotation péjorative, utilisé d'ailleurs dans la littérature de spécialité consacrée au bi- et au plurilinguisme, notamment par Georges Lüdi et Bernard Py: «[T] oute personne plongée dans un milieu géographique, culturel et linguistique nouveau, quelles que soient les raisons, les circonstances sociales et la durée de ce changement» (Lüdi et Py 2002: 18).

5. Père SAmuel (2010): Annuaire de l'Eglise Orthodoxe de France. Villebazy: Éditions du Monastère de Cantauque.

6. Nous rappelons que nous ferons référence ici exclusivement aux traductions faites en France, parmi lesquelles nous inclurons celle du père belge Denis Guillaume, largement utilisée dans de nombreuses paroisses de l'Hexagone.

7. Saint Jean Chrysostome (Iv s. $/ 1925)$ : La Divine Liturgie de notre Père Saint Jean Chrysostome. (Texte grec et traduction française avec introduction et notes par Dom Placide DE MEester) $3^{\mathrm{e}}$ édition revue et augmentée. Rome/Paris: Gabalda.

8. Saint Jean Chrysostome (IV s./s. d.): La Divine Liturgie de notre saint Père Jean Chrysostome. (Traduit par le père Placide Deseille) St-Laurent-en-Royans/Monastère Saint-Antoine-Le-Grand, métochion de Simonos Petra.

9. Nous comprenons ici le paratexte dans l'acception de Gérard Genette, comme l'un des cinq types de relations transtextuelles, relation entretenue par le texte proprement dit avec son titre, son sous-titre, sa préface, sa postface, son avertissement, son avant-propos, etc. (Genette 1982: 10).

10. Saint Jean Chrysostome et Saint Basile de Césarée (Iv ${ }^{e}$ s./2009): Les Divines Liturgies de saint Jean Chrysostome, de saint Basile le Grand et la Liturgie des Dons présanctifiés selon l'usage du Mont Athos. (Traduit par le père Placide Deseille) St-Laurent-en-Royans/La Bastide d'Engras: Éditions du Monastère Saint-Antoine-Le-Grand/Monastère de Solan. 
11. Comme dans le cas de la brochure qui l'a précédée, employée pour les célébrations (donc à fonctionnalité rituelle et liturgique, explicitée par les conditions de sa réalisation) dans les deux communautés monastiques déjà mentionnées.

12. LARCHET, Jean-Claude (2010): Recension: "Les divines liturgies de saint Jean Chrysostome, de saint Basile le Grand et la liturgie des dons présanctifiés», traduction nouvelle du père Placide Deseille. Accessible sur le site Web Orthodoxie - L'information orthodoxe sur Internet (Dernière mise à jour : 8 octobre 2012): Consulté le 20 juin 2013, <http://www.orthodoxie.com/2010/02/recension-lesdivines-liturgies-de-saint-jean-chrysostome-de-saint-basile-le-grand-et-la-liturgie-de.html>.

13. «Cette traduction de la liturgie qui est le plus souvent célébrée, celle de saint Jean Chrysostome, s'ajoute aux cinq traductions actuellement en usage dans les pays francophones: 1) celle de Mgr Sylvestre (Nice, 1965); 2) celle des éditions Liturgica; 3) celle du père Denis Guillaume (qui figure dans plusieurs volumes de ses publications, notamment dans un petit livre de poche qui regroupe les trois liturgies); 4) la traduction des moines de Cantauque (publiée avec la bénédiction de S. E. le métropolite Joseph); 5) la traduction réalisée et publiée par la Fraternité orthodoxe en 2007, reçue par la commission liturgique de l'Assemblée des évêques orthodoxes de France» (Larchet 2010; voir note 12).

14. Saint Jean Chrysostome (IV s./1981): Divine liturgie selon saint Jean Chrysostome. (Traduit par Jean Meyendorff et Boris Bobrinskoy) Paris: Liturgica.

15. Saint Jean Chrysostome (IV s./1986): Hiératikon. Tome 2. Les Divines Liturgies. (Traduit par Denis Guillaume [non formellement mentionné]) Rome: Diaconie Apostolique.

16. La plupart des orthodoxes bénéficiaires de ses traductions ont souvent critiqué des passages trop obscurs ou ambigus, appréciant plutôt l'effort d'ensemble du père Denis Guillaume, qui avait traduit l'intégralité des offices orthodoxes, et s'attardant moins à la qualité linguistique et stylistique de son travail de traducteur. Sans parler du fait qu'il était Belge, donc pas Français, seulement francophone.

17. Saint Jean Chrysostome (Iv $\left.{ }^{\mathrm{e}} \mathrm{s} . / 2002\right)$ : Divines liturgies de saint Jean Chrysostome et de saint Basile de Césarée. (Traduit par l'archimandrite Јасов, le hiéromoine EliséE et le père dr. Y. Goldman) Villebazy: Monastère de la Théotokos.

18. Saint Jean Chrysostome et Saint Basile de Césarée (Ive s./2002/2006): Divines liturgies de saint Jean Chrysostome et de saint Basile de Césarée. $2^{\mathrm{e}}$ édition corrigée et complétée. (Traduit par l'archimandrite ЈАСов, le hiéromoine EliséE et le père dr. Y. Goldman) Villebazy: Monastère de la Théotokos. Consulté le 12 décembre 2012, <http://www.monastere-cantauque.com/divine-liturgie-fr.pdf>.

19. Livre liturgique qui comprend les trois liturgies avec leurs prières, les formules de congé, etc. (Dumas 2010b: 125).

20. Saint Jean Chrysostome (IV s. 2007$)$ : La Divine Liturgie de saint Jean Chrysostome. (Traduit par l'Assemblée des évêques orthodoxes de France) Mayenne: Fraternité orthodoxe en Europe occidentale.

21. Site Web de la Fraternité orthodoxe en Europe occidentale. (Dernière mise à jour : 8 octobre 2012): Consulté le 20 juin 2012, <http://fraternite-orthodoxe.eu/qu_est_ce_que_la_fraternite_orthodoxe___034.htm>. L'un des membres fondateur de la Fraternité orthodoxe en Europe ocidentale est le père Boris Bobrinskoy.

22. Même si c'est elle qui est mentionnée en tant qu'auteure de la traduction: «traduction de l'Assemblée des évêques orthodoxes de France».

23. Saint Jean Chrysostome (IV s./2009): Les Divines Liturgies de saint Jean Chrysostome, de saint Basile le Grand et la Liturgie des Dons présanctifiés, selon l'usage du Mont Athos. Traductions publiées avec la bénédiction de son Eminence le Métropolite Emmanuel, métropolite grecorthodoxe de France, Patriarcat œcuménique. St-Laurent-en-Royans/La Bastide d'Engras: Éditions du Monastère Saint-Antoine-Le-Grand/Monastère de Solan.

24. Option qui, selon nous, rend compte de son humilité monastique.

25. Il s'agit, rappelons-le, de la seconde édition de la traduction française des liturgies de saint Jean Chrysostome et de saint Basile de Césarée publiée au monastère de Cantauque en 2006, ainsi que de la traduction des mêmes textes liturgiques publiée aux monastères Saint-Antoine-Le-Grand et de la Protection de la Mère de Dieu de Solan, en 2009. 


\section{RÉFÉRENCES}

Benveniste, Émile (1966): Problèmes de linguistique générale. Vol. I. Paris: Gallimard.

Dumas, Felicia (2000): Gest şi expresie în liturghia ortodoxă. Studiu semiologic [Expression des gestes dans la liturgie orthodoxe. Etude sémiologique]. Iași: Institutul European.

Dumas, Felicia (2009): L'orthodoxie en langue française. Perspectives linguistiques et spirituelles. Iași: Demiurg.

Dumas, Felicia (2010a) : Dicționar bilingv de termeni religioşi ortodocşi : român-francez. [Dictionnaire bilingue de termes religieux orthodoxes: roumain-français]. Iași : Doxologia.

Dumas, Felicia (2010b) : Dictionnaire bilingue de termes religieux orthodoxes: français roumain. Iași : Doxologia.

Dumas, Felicia (2011) : Marques lexicales d'une identité plurielle de l'Orthodoxie d'expression française. Synergies Italie. 7:51-61. Cracovie: Eikon Plus.

Evdokimov, Paul (1985): La Prière de l'Église d'Orient: la Liturgie byzantine de saint Jean Chrysostome. Paris: Desclées de Brouwer.

Genette, Gérard (1982): Palimpsestes - La littérature au second degré. Paris: Seuil.

LÜDI, Georges et Py, Bernard (2002): Être bilingue. $2^{e}$ édition révisée. Berne: Peter Lang.

Meschonnic, Henri (1999): Poétique du traduire. Lagrasse: Verdier.

Monti, Enrico (2012) : La retraduction, un état des lieux. In: Enrico Monti et Peter Schnyder, éd. Autour de la retraduction. Perspectives littéraires européennes. Paris: Orizons.

Oustinoff, Michaël (2011): Traduire et communiquer à l'heure de la mondialisation. Paris: CNRS Éditions.

PA procki, Henryk (1993): Le mystère de l'eucharistie, genèse et interprétation de la liturgie eucharistique byzantine. (Traduit du polonais par Françoise LHOEst) Paris: Cerf.

Pyм, Anthony (1997): Pour une éthique du traducteur. Arras/Ottawa: Artois Presses Universitél Presses de l'Université d'Ottawa.

\section{ANNEXE}

\section{Glossaire de termes religieux orthodoxes}

ANAPHORE (subst. fém.): Partie centrale des liturgies eucharistiques orthodoxes, qui correspond au canon eucharistique; c'est la prière centrale du sacrifice eucharistique, pendant laquelle les saints Dons sont consacrés.

ARCHIMANDRITE (subst. masc.): Titre honorifique accordé à un moine prêtre; le supérieur ou l'higoumène d'un monastère orthodoxe est choisi, en général, parmi les archimandrites.

ArкнiÉratikon (subst. masc.): Livre liturgique qui comprend des offices particuliers qui ne peuvent être célébrés que par les évêques, tels la chirotonie, la consécration des églises, etc.

Aтноnite (adj.): Relatif à la Sainte Montagne de l'Athos.

Euchologe (subst. masc.): Livre de prières à l'usage du prêtre, qui contient l'office de la liturgie eucharistique, les autres offices sacramentels, ainsi que toutes sortes de bénédictions et de prières.

HiÉRARQUe (subst. masc.): Chef ecclésiastique, nom porté par les évêques (archevêques, métropolites ou patriarches).

HiÉRATikon (subst. masc.): Livre liturgique qui comprend les règles et les textes des prières des prêtres et des diacres pour les divines liturgies, les vêpres et les matines, la liturgie pontificale, les prières de l'ambon, de la communion, etc.

Higoumène (subst. masc.): Supérieur d'un monastère orthodoxe masculin.

Hy mne (subst. fém.) : Composition patristique formée de plusieurs strophes régulières chantées sur une certaine mélodie; dans l'orthodoxie, il y a plusieurs types d'hymnes: les tropaires, les kondakia, les hirmi, les antiennes, etc.

MÉNÉEs (subst. fém. au pluriel) : Livres liturgiques, un pour chaque mois, qui comprennent les parties variables des offices célébrés lors des fêtes fixes de toute l’année liturgique. 
MÉTochion (subst. masc.; pluriel métochia): Dépendance d’un grand monastère, en général du Mont Athos.

Pannychide (subst. fém.): Livre qui comprend l'office de la pannychide (la grande et, plus abrégée, la petite), ainsi que le typikon des offices consacrés aux morts (les funérailles des moines, des prêtres, etc.).

Rubriques (subst. fém. au pluriel): Notes, généralement écrites en rouge, qui indiquent l'ordo, les règles du déroulement des célébrations liturgiques.

Triode (subst. masc.) : Appelé aussi Triode de carême, c'est le livre liturgique qui comprend les textes du Grand Carême, ainsi que ceux de la Semaine Sainte jusqu'au Samedi Saint.

Tүрікоn (subst. masc.): Ensemble de règles spirituelles et de déroulement de la vie monastique respectées par les membres d'une communauté monastique; rubriques qui indiquent l'ordo, les règles du déroulement des célébrations liturgiques.

UsAGE (subst. masc.): mode de déroulement des offices selon des règles établies par l’Église. La notion d'usage renvoie à la réalité pratique de la tradition ou des traditions liturgiques locales développées par les grandes églises autocéphales, notamment grecque et russe. Dans l'orthodoxie, on parle des usages grec et slave. 